\title{
Importancia de la hipertensión pulmonar relativa en la lesión renal aguda posterior al trasplante cardíaco
}

\author{
Juan C. Bianco
}

Bianco JC, Stang MV, Denault AY, Marenchino RG, Belziti CA, Musso CG. Acute Kidney Injury after Heart Transplant: The Importance of Pulmonary Hypertension. J Cardiothorac Vasc Anesth. 2021; 35:2052-2062. https://doi.org/10.1053/j.jvca.2020.12.010

La lesión (injuria) renal aguda (IRA) posterior al trasplante cardíaco es una complicación frecuente que se asocia con resultados clínicos adversos, incluida la disminución de la sobrevida a corto y largo plazo ${ }^{1}$. La incidencia de la IRA entre los pacientes sometidos a trasplante cardíaco se ha incrementado en los últimos años, oscilando entre el 40 y el $86 \%$ en estudios actuales ${ }^{1,2}$. La mayoría de los pacientes con insuficiencia cardíaca en estadio terminal, candidatos a trasplante cardíaco, padecen hipertensión pulmonar (HTP) y esta última enfermedad desempeña un papel clave en el síndrome cardiorrenal en el período perioperatorio del trasplante cardíaco ${ }^{3}$. El aumento de la resistencia vascular pulmonar (RVP) del receptor puede provocar que el ventrículo derecho del donante no se adapte al aumento súbito de la poscarga cuando se implanta el corazón del donante, desencadenando una falla aguda del ventrículo derecho. Para evaluar el impacto de la gravedad de la HTP en la IRA del trasplante cardíaco se han estudiado índices de HTP, tales como presiones arteriales pulmonares absolutas, RVP y gradientes de presiones pulmonares ${ }^{2}$. La HTP relativa definida por el cociente que relaciona la presión arterial media (PAM) con la presión arterial pulmonar media (PAPM) ofrece la ventaja de tener en cuenta la estrecha relación entre la circulación pulmonar y sistémica, y el impacto de la HTP sobre las presiones sistémicas; (PAM/ PAPM valor normal $>4)^{4,5}$. Aunque varias publicaciones han abordado el tema de IRA en el trasplante cardíaco, la importancia de la HTP relativa (PAM/PAPM) como predictor de IRA postrasplante cardíaco nunca ha sido estudiado. El objetivo primario del estudio fue determinar si la HTP relativa se asocia significativamente a la IRA grave postrasplante cardíaco.

Después de obtener la aprobación del Comité de Ética, se estudiaron retrospectivamente pacientes adultos consecutivos que fueron sometidos a trasplante cardíaco entre enero de 2009 y diciembre de 2017 en el Hospital Italiano de
Buenos Aires, con seguimiento hasta diciembre de 2019. Los pacientes incluidos en el estudio fueron aquellos sometidos al primer trasplante cardíaco ortotópico, con 18 años de edad o mayores. Los pacientes que se sometieron a un trasplante adicional de otros órganos (riñón, hígado, pulmón) antes del trasplante cardíaco o simultáneamente a él, y aquellos que murieron dentro de las 24 horas posteriores al trasplante cardíaco fueron excluidos. Un catéter de termodilución para arteria pulmonar fue introducido a los pacientes para obtener un perfil hemodinámico perioperatorio completo, como parte de la evaluación pretrasplante y en el posoperatorio inmediato, al llegar a Unidad de Cuidados Intensivos Cardíacos.

De acuerdo con los criterios $\mathrm{KDIGO}^{6}$, se definió IRA y se estratificaron los pacientes según el estadio de gravedad alcanzado dentro de los 7 días posteriores al trasplante cardíaco. Los pacientes se dividieron en 2 grupos basados en el desarrollo de IRA grave (estadio 3 ) en el posoperatorio del trasplante cardíaco.

El estudio incluyó a 184 pacientes adultos sometidos a trasplante cardíaco. Entre ellos, el 83,2\% sufrió IRA, incluyendo $40,8 \%, 19,6 \%$ y $22,8 \%$ en IRA estadios 1,2 y 3 , respectivamente. Veintinueve pacientes $(15,8 \%)$ requirieron terapia de reemplazo renal en el posoperatorio. La edad mediana de los pacientes receptores fue de 54 (45-61) años; 74,5\% fueron de sexo masculino. Distintos factores de riesgo se relacionaron significativamente con el desarrollo de IRA grave postrasplante cardíaco en el análisis univariado, incluyendo factores de riesgo preoperatorios como la mayor edad, los antecedentes de insuficiencia hepática y cirugía cardíaca, la menor tasa de filtrado glomerular estimada (TFGe), la HTP relativa, una mayor presión venosa central (PVC) y PAPM y un menor índice de pulsatilidad arterial pulmonar. Además, se identificaron varios factores de riesgo intraoperatorios y posoperatorios, tales como el mayor número de unidades 
de productos sanguíneos transfundidas, la disfunción del ventrículo derecho, la disfunción primaria del injerto, la menor fracción de eyección del ventrículo izquierdo, las infecciones y la insuficiencia hepática. La HTP relativa (PAM/PAPM) fue el único parámetro hemodinámico que estuvo significativamente asociado a IRA postrasplante cardíaco tanto en el preoperatorio $(2,1[1,8-2,9]$ vs. 2,4 $[2,0-3,3] ; \mathrm{p}=0,016)$, como en el posoperatorio $(2,5[2,3-$ $3,2]$ vs. $3,1[2,5-3,8], \mathrm{p}=0,005)$.

La IRA estadio 3 se asoció significativamente con un incremento de la mortalidad a los 30 días (Hazard Ratio [HR]: 10,58, intervalo de confianza [IC] 95\%: 3,37-33,28; $\mathrm{p}<0,001$ ), 1 año (HR: 3,55; IC 95\%: 1,69-7,47; $\mathrm{p}<0,001$ ), 5 años (HR: 2,10; IC 95\%: 1,14-3,83; $p=0,014$ ) y hasta 8 años (HR: 1,88 ; IC 95\%: 1,03-3,40; $p=0,035$ ).

En el análisis multivariado, los factores independientes relacionados con la IRA grave tras el trasplante cardíaco fueron la HTP relativa preoperatoria (Odds Ratio [OR]: 1,62; IC 95\%: 1,05-2,49; $\mathrm{p}=0,028$ ), la relación PVC/ presión capilar pulmonar preoperatoria (OR: 3,59; IC 95\%: $1,13-11,43 ; p=0,030)$ y la disfunción ventricular derecha posoperatoria (OR: 3,63; IC 95\%: 1,50-8,75; $\mathrm{p}=0,004$ ). Por el contrario, la TFGe preoperatoria (OR: 0,99; IC 95\%: $0,97-1,00 ; p=0,143$ ) no se relacionó con la IRA grave.

En este estudio de pacientes sometidos a trasplante cardíaco hemos observado que algunas variables relacionadas con la hemodinamia preoperatoria y posoperatoria se asociaron con IRA grave. Estas incluyeron HTP relativa, relación de presiones auriculares derecha e izquierda y disfunción del ventrículo derecho. La TFGe preoperatoria no se relacionó con la IRA grave postrasplante cardíaco.

La HTP está presente frecuentemente en pacientes con insuficiencia cardíaca terminal, candidatos para trasplante cardíaco, y es un factor de riesgo importante para una mayor morbilidad y mortalidad ${ }^{7,8}$. Los pacientes con HTP se consideran de alto riesgo para desarrollar falla del ventrículo derecho al momento de desconectarlos de la circulación extracorpórea y en el período posoperatorio temprano. Por su parte, el corazón del donante no está acostumbrado a una RVP elevada y, por lo tanto, es susceptible de padecer una falla aguda del ventrículo derecho al momento del implante en un receptor con HTP. La insuficiencia ventricular derecha se caracteriza por una disminución en el volumen sistólico del ventrículo derecho que conduce a un llenado insuficiente del ventrículo izquierdo. El llenado del ventrículo izquierdo también se ve afectado debido al desplazamiento del tabique interventricular hacia la izquierda. Esto, junto con la disfunción sistólica/diastólica del ventrículo derecho, contribuye a la reducción del gasto cardíaco y, por lo tanto, una disminución de la perfusión renal ${ }^{9}$. Como la falla del ventrículo derecho y la HTP aumentan la presión auricular derecha o PVC, también pueden aumentar el riesgo de síndrome cardiorrenal por congestión venosa. El aumento de la PVC conduce a una reducción de la presión de perfusión renal, deteriora el flujo sanguíneo intrarrenal y activa el sistema renina-angiotensina-aldosterona y el sistema nervioso simpático, lo que lleva a una reducción de la $\mathrm{TFG}^{10}$. El reconocimiento del síndrome cardiorrenal en el contexto perioperatorio del trasplante cardíaco puede brindar la posibilidad de aplicar estrategias de prevención y tratamiento. En particular, la predicción temprana de IRA posoperatoria basada en la HTP relativa preoperatoria y su posterior disfunción del ventrículo derecho podría ayudar a realizar una intervención proactiva y oportuna en los pacientes con alto riesgo. Por consiguiente, se requieren estudios adicionales para determinar en qué medida la HTP podría ser reversible y representaría una posibilidad de tratamiento potencial para prevenir o mitigar la gravedad de la IRA postrasplante cardíaco.

Conflictos de interés: el autor declara no tener conflictos de interés.

\section{REFERENCIAS}

1. Thongprayoon $\mathrm{C}$, Lertjitbanjong $\mathrm{P}$, Hansrivijit P, et al. Acute Kidney Injury in Patients Undergoing Cardiac Transplantation: A Meta-Analysis. Medicines (Basel). 2019; 6(4):108.

2. Guven G, Brankovic M, Constantinescu AA, et al. Preoperative right heart hemodynamics predict postoperative acute kidney injury after heart transplantation. Intensive Care Med. 2018; 44(5):588-97.

3. Miller WL, Grill DE, Borlaug BA. Clinical features, hemodynamics, and outcomes of pulmonary hypertension due to chronic heart failure with reduced ejection fraction: pulmonary hypertension and heart failure. JACC Heart Fail. 2013; 1(4):290-9.
4. Bianco JC, Qizilbash B, Carrier M, et al. Is patient-prosthesis mismatch a perioperative predictor of long-term mortality after aortic valve replacement? J Cardiothorac Vasc Anesth. 2013; 27(4):647-53.

5. Robitaille A, Denault AY, Couture P, et al. Importance of relative pulmonary hypertension in cardiac surgery: the mean systemic-topulmonary artery pressure ratio. J Cardiothorac Vasc Anesth. 2006; 20(3):331-9.

6. Kidney Disease Improving Global Outcomes (KDIGO) Acute Kidney Injury Work Group. KDIGO clinical practice guideline for acute kidney injury. Kidney Int Suppl. 2012;2:1-138.

7. Bianco JC, Mc Loughlin S, Denault AY, et al. Heart Transplantation in Patients $>60$ Years: Importance of Relative Pulmonary Hypertension and Right Ventricular Failure on Midterm Survival. J Cardiothorac Vasc Anesth. 2018; 32(1):32-40.

8. Sobieszczańska-Małek M, Zieliński T, Piotrowski W, et al. Prognostic value of pulmonary hemodynamic parameters in cardiac transplant candidates. Cardiol J. 2014; 21(5):532-8

9. Schrier RW, Bansal S. Pulmonary hypertension, right ventricular failure, and kidney: different from left ventricular failure? Clin J Am Soc Nephrol. 2008; 3(5):1232-7. 10. Damman K, Voors AA, Navis G, et al. The cardiorenal syndrome in heart failure. Prog Cardiovasc Dis. 2011; 54(2):144-53. 